\title{
A NEW PROOF OF N. J. YOUNG'S THEOREM ON THE ORBITS OF THE ACTION OF THE SYMPLECTIC GROUP
}

\author{
by DAN TIMOTIN
}

(Received 11th July 1995)

\begin{abstract}
The group of symplectic transformations acts on the unit ball of a Hilbert space. The structure of the orbits has been determined by $\mathbf{N}$. J. Young in [8]. We provide a new proof of this theorem; it is slightly simpler than the original one, and does not involve Brown-Douglas-Fillmore theory. Moreover, the steps followed hopefully throw some additional light on the subject. We rely heavily on previous work of Khatskevich, Shmulyan and Shulman $([5,6,7)$; the proofs of the results used are included for completeness.
\end{abstract}

1991 Mathematics subject classification: 47A99.

\section{Young's theorem}

Suppose $\mathcal{H}, \mathcal{K}$ are Hilbert spaces, and let $\mathcal{B}$ denote the closed unit ball of $\mathcal{L}(\mathcal{H}, \mathcal{K})$. The problem considered by Young in [8] is that of characterizing the orbit of the action of the symplectic group on $\mathcal{B}$. The symplectic group $G$ is defined as the group of all $2 \times 2 J$-unitary operator matrices; that is, all invertible matrices $g=\left(\begin{array}{cc}A & B \\ C & D\end{array}\right)$, $A \in \mathcal{L}(\mathcal{K}), B \in \mathcal{L}(\mathcal{H}, \mathcal{K}), C \in \mathcal{L}(\mathcal{K}, \mathcal{H}), D \in \mathcal{L}(\mathcal{H})$ which satisfy the relation

$$
\left(\begin{array}{cc}
A & B \\
C & D
\end{array}\right)^{-1}=\left(\begin{array}{cc}
A^{*} & -C^{*} \\
-B^{*} & D^{*}
\end{array}\right)
$$

The action of $G$ on $\mathcal{B}$ is given by the maps

$$
\Phi_{g}(X)=(A X+B)(C X+D)^{-1} \text {. }
$$

On the open unit ball (the set of all strict contractions) $G$ acts transitively. This is no more the case on $\mathcal{B}$; the orbits of the action have a rather complicated structure, which is determined by Young's result.

Theorem 1. $X$ and $Y$ lie in the same orbit if and only if the following conditions are satisfied: 
(i) there exist invertible operators $P$ and $Q$, such that

$$
I-Y^{*} Y=P^{*}\left(I-X^{*} X\right) P, I-Y Y^{*}=Q^{*}\left(I-X X^{*}\right) Q
$$

(ii) if $X$ is essentially unitary (and thus also $Y$ by (1)), then

$$
\text { ind } X=\text { ind } Y \text {. }
$$

Conditions (i) are simple consequences of the identities:

$$
\begin{aligned}
& I-\Phi_{g}(X)^{*} \Phi_{g}(X)=\left(X^{*} C^{*}+D^{*}\right)^{-1}\left(I-X^{*} X\right)(C X+D)^{-1} \\
& I-\Phi_{g}(X) \Phi_{g}(X)^{*}=\left(X B^{*}+A^{*}\right)^{-1}\left(I-X X^{*}\right)\left(B X^{*}+A\right)^{-1}
\end{aligned}
$$

The necessity of (ii) is less apparent and will be shown later.

Remark. It should be mentioned that Theorem 1 has been independently obtained, in the different context of Krein spaces, by Azizov ([2]). A readable exposition can be found in [3].

\section{Preliminary facts}

We will use repeatedly the following simple lemma (see, for instance, [4]).

Lemma 1 (a) For $A, B$ bounded operators, the following statements are equivalent.

(i) There exists $X$ bounded, such that $A=X B$.

(ii) There exists $Y$ bounded, such that $A^{*} A=B^{*} Y B$.

(iii) range $A^{*} \subset$ range $B^{*}$.

(iv) $A^{*} A \leq c B^{*} B$ for some constant $c>0$.

(b) If $Y$ is invertible in (ii), then we also have $B=X^{\prime} A$ for some bounded $X^{\prime}$.

For any contraction $T$, the symbol $D_{T}$ will denote the defect operator $\left(I-T^{*} T\right)^{1 / 2}$; we have thus $D_{T^{*}}=\left(I-T T^{*}\right)^{1 / 2}$. As customary, for any operator $T,|T|=\left(T^{*} T\right)^{1 / 2}$

The next result is a structure theorem for $J$-unitary $2 \times 2$ operator matrices. It seems to have been several times rediscovered; a good reference is [1].

Theorem 2. For any J-unitary operator matrix $g=\left(\begin{array}{ll}A & B \\ C & D\end{array}\right)$ there exist a strict contraction $T \in \mathcal{L}(\mathcal{H}, \mathcal{K})(\|T\|<1)$ and unitary operators $U_{1} \in \mathcal{L}(\mathcal{K}), U_{2} \in \mathcal{L}(\mathcal{H})$ such that 


$$
\left(\begin{array}{ll}
A & B \\
C & D
\end{array}\right)=\left(\begin{array}{cc}
U_{1} & 0 \\
0 & U_{2}
\end{array}\right)\left(\begin{array}{cc}
-D_{T^{*}}^{-1} & T D_{T}^{-1} \\
-T^{*} D_{T^{*}}^{-1} & D_{T}^{-1}
\end{array}\right) .
$$

As a consequence, we have, after a few computations

$$
\Phi_{g}(X)=U_{1} \phi_{T}(X) U_{2}^{*}
$$

where

$$
\phi_{T}(X)=T-D_{T} \cdot X\left(I-T^{*} X\right)^{-1} D_{T}
$$

Note also, for further reference, that relations (3) and (4) become

$$
\begin{aligned}
& I-\phi_{T}(X)^{*} \phi_{T}(X)=D_{T}\left(I-X^{*} T\right)^{-1}\left(I-X^{*} X\right)\left(I-T^{*} X\right)^{-1} D_{T} \\
& I-\phi_{T}(X) \phi_{T}(X)^{*}=D_{T^{*}}\left(I-X T^{*}\right)^{-1}\left(I-X X^{*}\right)\left(I-T X^{*}\right)^{-1} D_{T^{*}}
\end{aligned}
$$

Actually, formula (6) makes sense in an even larger context, namely when $T$ and $X$ are two contractions subjected to the condition that $I-T^{*} X$ should be invertible. Obviously in this case $I-X T^{*}$ is also invertible, and equations (7) and (8) remain valid.

Formula (5) suggests the introduction of some ad-hoc terminology. We will call two contractions $X$ and $Y$ orthogonally equivalent, and write $X \simeq Y$, iff there exist unitary operators $U \in \mathcal{L}(\mathcal{K})$ and $V \in \mathcal{L}(\mathcal{H})$ such that $Y=U X V$. Note that this is indeed an equivalence relation on $\mathcal{B}$ (in [6] it is called a congruence). According to formula (5), the orbit of $X$ coincides with the set of all contractions orthogonally equivalent to an operator of the form $\phi_{T}(X)$, with $\|T\|<1$. The following simple lemma gives a useful criterion.

Lemma ([6]). If $|X|$ is unitary equivalent to $|Y|$ and $\left|X^{*}\right|$ is unitarily equivalent to $\left|Y^{*}\right|$, then $X \simeq Y$.

Proof. If $|X|=U^{*}|Y| U$, then the map $X h \mapsto Y U h$ is an isometric map from range $X$ to range $Y$. The second condition insures that it can be extended to a unitary $V$, since the orthogonal complements of the ranges (which coincide with ker $\left|X^{*}\right|$ and ker $\left.\left|Y^{*}\right|\right)$ have the same dimensions. Then $X=V^{*} Y U$.

\section{A symmetry formula}

There is a hidden symmetry between $T$ and $X$ which is not apparent in the definition of $\phi_{T}(X)$. This is shown by the following result from [6].

Theorem 3. If $\|T\|<1$, and $\|X\| \leq 1$, then $\phi_{T}(X) \simeq \phi_{X}(T)$. 
Proof. Denote $S=D_{X}\left(I-T^{*} X\right)^{-1} D_{T}$. A direct computation shows that

$$
\begin{aligned}
& \phi_{T}(X)^{*} \phi_{T}(X)=I-S^{*} S, \\
& \phi_{X}(T)^{*} \phi_{X}(T)=I-S S^{*} .
\end{aligned}
$$

Since $D_{T}$ is invertible, $\operatorname{dim} \operatorname{ker} S=\operatorname{dim} \operatorname{ker} S^{*}=\operatorname{dim} \operatorname{ker} D_{X}$. The polar decomposition of $S$ implies then that $S^{*} S$ and $S S^{*}$ are unitarily equivalent, and thus also $\phi_{T}(X)^{*} \phi_{T}(X)$ and $\phi_{X}(T)^{*} \phi_{X}(T)$. A similar argument shows that $\phi_{T}(X) \phi_{T}(X)^{*}$ and $\phi_{X}(T) \phi_{X}(T)^{*}$ are unitarily equivalent; the theorem follows by Lemma 2 .

Note that in [6] an example is given of two contractions (neither one strict), for which $I-T^{*} X$ is invertible, consequently $\phi_{T}(X)$ and $\phi_{X}(T)$ both can be defined, but are not orthogonally equivalent.

In this moment we may already prove the remaining assertion in the proof of the necessity of Young's conditions. Indeed, if $D_{X}^{2}$ is compact, then $D_{X}$ is also compact. If $Y=\phi_{X}(T)=X-D_{X^{*}} T\left(I-X^{*} T\right)^{-1} D_{X}$, it follows that $Y-X$ is compact and therefore ind $X=$ ind $Y$. Furthermore, the index is invariant with respect to orthogonal equivalence; therefore formula (5) and Theorem 3 yield (2).

\section{Shmulyan's equivalence}

In [7] Shmulyan introduces the following equivalence relation on $\mathcal{B}: X$ and $Y$ are said to be S-equivalent if and only if there exist bounded operators $A$ and $B$ such that

$$
Y-X=D_{X^{*}} A D_{X}=D_{Y^{*}} B D_{Y}
$$

We will write $X \cong Y$ in this case. Obviously $X \cong Y$ implies $X^{*} \cong Y^{*}$. Also, note that the operators $A$ and $B$ can be chosen such that $\operatorname{ker} D_{X} \subset \operatorname{ker} A, \operatorname{ker} D_{X^{*}} \subset \operatorname{ker} A^{*}$, $\operatorname{ker} D_{Y} \subset \operatorname{ker} B, \operatorname{ker} D_{Y^{*}} \subset \operatorname{ker} B^{*}$; we will always suppose that this is achieved.

If $Y=X+D_{X^{*}} A D_{X}$, a simple computation shows that

$$
D_{Y}^{2}=D_{X}\left[\left(I-A^{*} X\right)\left(I-X^{*} A\right)-A^{*} A\right] D_{X} .
$$

By Lemma 1, range $D_{Y} \subset$ range $D_{X}$. By symmetry considerations we have that, if $X \cong Y$, then

$$
\text { range } D_{X}=\text { range } D_{Y}, \quad \text { range } D_{X^{*}}=\text { range } D_{Y^{*}}
$$

It is obvious that all strict contractions are S-equivalent, since their defect operators are invertible. Also, the following lemma is immediate.

Lemma 3. If $X \cong Y$ and $X^{\prime} \cong Y^{\prime}$, then $X \oplus X^{\prime} \cong Y \oplus Y^{\prime}$. 
More interesting is the following theorem, proved in [7]:

Theorem 4. The set $\left\{\phi_{X}(T) \mid\|T\|<1\right\}$ coincides with the $S$-equivalence class of $X$.

Proof. Suppose first that $\|T\|<1$. By the definition of $Y=\phi_{X}(T)$ we have already obtained one of the equalities in (9). On the other hand, since $D_{T}$ and $D_{T}^{2}$ are invertible, relations (7) and (8) (with $T$ and $X$ interchanged) and Lemma 1(b) show that there exist bounded operators $A$ and $B$, such that $D_{X}=A D_{Y}, D_{X^{*}}=D_{Y} . B$. Then

$$
X=Y-D_{Y^{*}} B T\left(I-X^{*} T\right)^{-1} A D_{Y}
$$

and the second equality in (9) is obtained.

Suppose now that $Y \cong X$. We have first $Y=X+D_{X} \cdot A D_{X}$ for some bounded operator $A$; note that $\operatorname{ker} D_{X} \subset \operatorname{ker} A$ and $\operatorname{ker} D_{X^{*}} \subset \operatorname{ker} A^{*}$.

Denote $W=I-X^{*} A$. By $(10)$, we have $D_{Y}^{2}=D_{X}\left(W^{*} W-A^{*} A\right) D_{X}$. Obviously then $W^{*} W-A^{*} A$ is positive on the range of $D_{X}$. If $h \in \operatorname{ker} D_{X}, A h=0, W h=h$, while $W^{*} h=\left(I-A^{*} X\right) h$. But $X$ maps $\operatorname{ker} D_{X}$ into $\operatorname{ker} D_{X^{*}} ;$ since $\operatorname{ker} D_{X^{*}} \subset \operatorname{ker} A^{*}, W^{*} h=h$, and thus $\left(W^{*} W-A^{*} A\right) h=h$. Thus, $W^{*} W-A^{*} A \geq 0$.

Now, by (11) and Lemma 1 , it follows that $D_{Y}^{2} \geq c D_{X}^{2}$ for some $c>0$. Then

$$
\left\langle\left(W^{*} W-A^{*} A\right) D_{X} h, D_{X} h\right\rangle=\left\langle D_{Y} h, D_{Y} h\right\rangle \geq c\left\langle D_{X} h, D_{X} h\right\rangle .
$$

Since we have shown above that $\left(W^{*} W-A^{*} A\right) h=h$ for $h \in \operatorname{ker} D_{X}$, it follows that

$$
W^{*} W-A^{*} A \geq c I \text {. }
$$

Define then an operator $T$ by $T W h=-A h$ on the range of $W$ and $T=0$ on the rest. Relation (12) shows that $T$ is a strict contraction. We have $-A=T W=T-T X^{*} A$ and thus $-\left(I-T X^{*}\right) A=T, \quad A=-\left(I-T X^{*}\right)^{-1} T . \quad$ Then $Y=X-D_{X^{*}}\left(I-T X^{*}\right)^{-1} T D_{X}=\phi_{X}(T)$, and the theorem is completely proved.

Remark. In [5] it is proved that Shmulyan's equivalence relation has an interesting geometrical interpretation as the facial relation of equivalence on the unit ball of the Hilbert space $\mathcal{H}$.

\section{Completing the proof}

The last result suggests that we introduce a notation for the equivalence relation obtained by "composing" orthogonal equivalence with Shmulyan's equivalence. Thus, $X \sim Y$ will mean that there exists $Z$ such that $X \simeq Z$ and $Z \cong Y$. It can be seen directly that $\sim$ is an equivalence relation; however, in our case this follows from the fact that the set $\{Y \mid Y \sim X\}$ is just the orbit $X$ under the action of the symplectic group. This fact (first proved in [6]) follows from formula (5) and Theorems 3 and 4. 
Thus, to complete the proof of Young's theorem, we have to show that conditions (i) and (ii) imply that $Y \sim X$.

We begin with the following lemma (compare Lemma 2.17 of [5]).

Lemma 4. If (1) is satisfied, then $|X|$ is unitarily equivalent to an operator S-equivalent to $|Y|$.

Proof. Suppose $D_{Y}^{2}=P^{*} D_{X}^{2} P$. Thus, $\left\|D_{Y} \xi\right\|=\left\|D_{X} P \xi\right\|$ for any $\xi \in \mathcal{H}$; since $P$ is invertible, we may define an isometry $\Omega: \mathcal{D}_{Y} \rightarrow \mathcal{D}_{X}$, such that

$$
D_{X}=\Omega D_{Y} P^{-1} \text {. }
$$

But $\mathcal{H} \ominus \mathcal{D}_{Y}=\operatorname{ker} D_{Y}=\left\{\xi \mid P \xi \in \operatorname{ker} D_{X}\right\}$; using again the invertibility of $P$, it follows that $\operatorname{dim} \operatorname{ker} D_{Y}=\operatorname{dim} \operatorname{ker} D_{X}$, and thus $\Omega$ can be extended to a unitary that we will denote by the same letter, and still satisfying (13). Then

$$
\begin{aligned}
(I-|X|)^{1 / 2} & =(I+|X|)^{-1 / 2} D_{X}=(I+|X|)^{-1 / 2}\left(P^{*}\right)^{-1}(I+|Y|)^{1 / 2}(I-|Y|)^{1 / 2} \Omega^{*} \\
& =Q(I-|Y|)^{1 / 2} \Omega^{*}
\end{aligned}
$$

where $Q$ is again invertible. Thus

$$
I-|X|=\Omega(I-|Y|)^{1 / 2} Q^{*} Q(I-|Y|)^{1 / 2} \Omega^{*}
$$

If we denote $X^{\prime}=\Omega^{*}|X| \Omega$, then

$$
\begin{aligned}
|Y|-X^{\prime} & =I-X^{\prime}-(I-|Y|)=(I-|Y|)^{1 / 2}\left(Q^{*} Q-I\right)(I-|Y|)^{1 / 2} \\
& =D_{|Y|}(I+|Y|)^{-1 / 2}\left(Q^{*} Q-I\right)(I+|Y|)^{-1 / 2} D_{|Y|} .
\end{aligned}
$$

This yields one of the relations in (9). The other may be obtained by similar computations, starting, instead of (13), with

$$
D_{Y}=\Omega^{*} D_{X} P \text {. }
$$

Unitary equivalence obviously implies orthogonal equivalence. But we have to obtain results about the operators themselves, not about their moduli.

Lemma 5. If

$$
\operatorname{dim} \operatorname{ker} X-\operatorname{dim} \operatorname{ker} X^{*}=\operatorname{dim} \operatorname{ker} Y-\operatorname{dim} \operatorname{ker} Y^{*}
$$

then $|X| \sim|Y|$ implies $X \sim Y$. 
Proof. If $\operatorname{dim} \operatorname{ker} X=\operatorname{dim} \operatorname{ker} X^{*}$, then $X=U|X|$ for a unitary operator $U$, and thus $X \simeq|X|$. By relation (14), the same is true for $Y$, and the lemma is thus proved.

If this does not happen, suppose, for instance, that $d=\operatorname{dim} \operatorname{ker} X-\operatorname{dim} \operatorname{ker} X^{*} \geq 0$. If $Z$ denotes the zero operator with range $\{0\}$ and domain a space of dimension $d$, then there exist unitary operators $U$ and $V$, such that $X$ is unitarily equivalent to $U|X| \oplus Z$, while $Y$ is unitarily equivalent to $V|Y| \oplus Z$. Lemma 3 yields then the desired result.

Lemma 5 takes care of the case when $X$ is essentially unitary. To conclude the proof of Young's theorem, suppose that, for instance, $I-X^{*} X$ is not compact. If $E$ is the spectral measure of $|T|$, it follows that there exists $r<1$, such that $E([0, r])$ is infinite dimensional. If $E^{\prime}=I-E([0, r])$, then there exists an isometry $\Omega_{1}: E^{\prime} \mathcal{H} \rightarrow \mathcal{H}$, with infinite dimensional cokernel, such that $\Omega_{1}|X|\left|E^{\prime} \mathcal{H}=X\right| E^{\prime} \mathcal{H}$. Let then $\Omega$ be any unitary on $\mathcal{H}$ which coincides with $\Omega_{1}$ on $E^{\prime} \mathcal{H}$. We claim that $\Omega|X| \cong X$. Indeed, $\Omega|X|$ is unitarily equivalent to $X \mid E^{\prime} \mathcal{H} \oplus X_{1}$, while $X$ is unitarily equivalent to $X \mid E^{\prime} \mathcal{H} \oplus X_{2}$, with $X_{1}, X_{2}$ strict contractions. Again Lemma 3, together with the fact that all strict contractions are S-equivalent yields the result.

Since neither $I-Y^{*} Y$ is compact, it follows that the same is true for $Y$. Thus $X \sim|X|$ and $Y \sim|Y|$; an application of Lemma 4 ends then the proof of Young's theorem.

\section{REFERENCES}

1. T. ANDo, Linear operators in Krein spaces (Lecture notes, Hokkaido University, 1979).

2. T. YA. Azizov, On the theory of extensions of isometric and symmetric operators in spaces with an indefinite metric (Russian), Dep., VINITI (1982), 3420-82,29.

3. T. YA. AzIzov and I. S. IoKhvidov, Linear operators in spaces with an indefinite metric (John Wiley, 1989).

4. R. G. Douglas, On majorization, factorization and range inclusion of operators on Hilbert space, Proc. Amer. Math. Soc. 17 (1966), 413-415.

5. V. A. Khatskevich, Yu. L. Shmulyan and V. S. Shulman, Preorders and equivalence on operator balls (Russian), Sibirsk. Mat. Zh. 32 (1991), 172-183.

6. V. A. Khatskevich, Yu. L. Shmulyan and V. S. Shulman, Orbits of the symplectic group and facial equivalence (Russian), Sibirsk. Mat. Zh. 33 (1992), 186-193.

7. YU. L. Shmulyan, Generalized linear-fractional transformations of operator balls (Russian), Sibirsk. Mat. Zh. 21 (1980), 114-131.

8. N. J. YounG, Orbits of the unit sphere of $\mathcal{L}(\mathcal{H}, \mathcal{K})$ under symplectic transformations, $J$. Operator Theory 11 (1984), 171-191.

Institute of Mathematics of the Romanian ACADEmy

P.O. BOX 1-764

BUCHAREST 70700

ROMANIA 